\title{
Pressure controlled trimerization for switching of anomalous Hall effect in triangular antiferromagnet $\mathrm{Mn}_{3} \mathrm{Sn}$
}

\author{
Charanpreet Singh, ${ }^{1}$ Vikram Singh $\odot,{ }^{2}$ Gyandeep Pradhan, ${ }^{1}$ Velaga Srihari $\odot,{ }^{3}$ Himanshu Kumar Poswal, ${ }^{3}$ Ramesh Nath, ${ }^{2}$ \\ Ashis K. Nandy $\mathbb{C}^{1,}{ }^{1, *}$ and Ajaya K. Nayak ${ }^{1}{ }^{1, \dagger}$ \\ ${ }^{1}$ School of Physical Sciences, National Institute of Science Education and Research, HBNI, Jatni 752050, India \\ ${ }^{2}$ School of Physics, Indian Institute of Science Education and Research Thiruvananthapuram, Kerala 695551, India \\ ${ }^{3}$ High Pressure and Synchrotron Radiation Physics Division, Bhabha Atomic Research Centre, Mumbai 400085, India
}

(Received 10 August 2020; revised 17 November 2020; accepted 18 November 2020; published 14 December 2020)

\begin{abstract}
Here, we present a detailed theoretical and experimental study on the pressure induced switching of the anomalous Hall effect (AHE) in the triangular antiferromagnetic (AFM) compound $\mathrm{Mn}_{3} \mathrm{Sn}$. Our theoretical model suggests pressure driven significant splitting of the in-plane $\mathrm{Mn}$ bond lengths, i.e., an effective trimerization, which in turn stabilizes a helical AFM ground state by modifying the interplane exchange parameters in the system. We experimentally demonstrate that the AHE in $\mathrm{Mn}_{3} \mathrm{Sn}$ reduces from $5 \mu \Omega \mathrm{cm}$ at ambient pressure to zero at an applied pressure of about 1.5 GPa. Furthermore, our pressure dependent magnetization study reveals that the conventional triangular AFM ground state of $\mathrm{Mn}_{3} \mathrm{Sn}$ systematically transforms into the helical AFM phase where the symmetry does not support a nonvanishing Berry curvature required for the realization of a finite AHE. The pressure dependent x-ray diffraction study rules out any role of structural phase transition in the observed phenomenon. In addition, the temperature dependent in-plane lattice parameter at ambient pressure is found to deviate from the monotonic behavior when the system enters into the helical AFM phase, thereby supporting the proposed impact of trimerization in controlling the AHE. We believe that the present study makes an important contribution toward understanding the stabilization mechanism of different magnetic ground states in $\mathrm{Mn}_{3} \mathrm{Sn}$ and related materials for their potential applications pertaining to AHE switching.
\end{abstract}

DOI: 10.1103/PhysRevResearch.2.043366

\section{INTRODUCTION}

Effective manipulation of magnetic structure can decisively control the electrical [1-5], thermal [6], and optical [7-9] properties in magnetic materials. In the case of electrical transport in a magnetic system, the spins of the conduction electrons exhibit a strong Hund's coupling with the underlying magnetic structure. The fictitious magnetic field arising from such an interaction can significantly modify the path of the conduction electrons, leading to the observation of the anomalous Hall effect (AHE) and topological Hall effect (THE) in ferromagnets [1] and antiferromagnets [3,4,10]. A lot of effort has been put forward to study the possibility of switching these transport signals to facilitate device applications. Although the AHE in ferromagnets has a great potential for its utilization as a memory element in spintronic devices, the only viable mechanism seems to be the reversal of the underlying magnetic structure. In this regard, antiferromagnets with diverse magnetic structures are excellent candidates

\footnotetext{
*aknandy@niser.ac.in

†ajaya@niser.ac.in
}

Published by the American Physical Society under the terms of the Creative Commons Attribution 4.0 International license. Further distribution of this work must maintain attribution to the author(s) and the published article's title, journal citation, and DOI. to achieve control over the AHE by tuning the magnetic ground state. The fact that antiferromagnets are extremely stable against external perturbations makes them attractive candidates for spintronics. Although electrical switching of antiferromagnets has been demonstrated recently $[11,12]$, the realization of such a scenario in real devices remains far.

In particular, the noncollinear antiferromagnets are extensively pursued as their magnetic states can be easily manipulated by spin transfer torque related mechanisms. Recently, the hexagonal crystal structure based triangular antiferromagnets $\mathrm{Mn}_{3} \mathrm{Sn}$ and $\mathrm{Mn}_{3}$ Ge have received special attention due to their various anomalous transport properties, both in bulk systems $[4,5,13,14]$ and thin films [15-17]. In the hexagonal structure of $\mathrm{Mn}_{3} \mathrm{Sn}$, the $\mathrm{Mn}$ atoms form a kagome-type lattice with $\mathrm{Sn}$ atoms residing at the center of the hexagon. The geometrical frustration arising due to the antiferromagnetic (AFM) coupling of Mn moments and the kagome type of arrangement of $\mathrm{Mn}$ atoms incite a $120^{\circ}$ magnetic ground state [18]. In this structure, two layers of $\mathrm{Mn}$ atoms in a unit cell together form an inverse triangular spin structure as depicted in Fig. 1(a). The topological Weyl semimetallic nature of these materials has been demonstrated by theoretical calculations [19,20], as well as by experimental studies [21]. The presence of Weyl nodes near the Fermi level is believed to be the source of large AHE in these materials [20-22]. These Weyl nodes occur in pairs with opposite chirality and act as magnetic monopoles in the momentum space. An electron crossing between two Weyl nodes of 

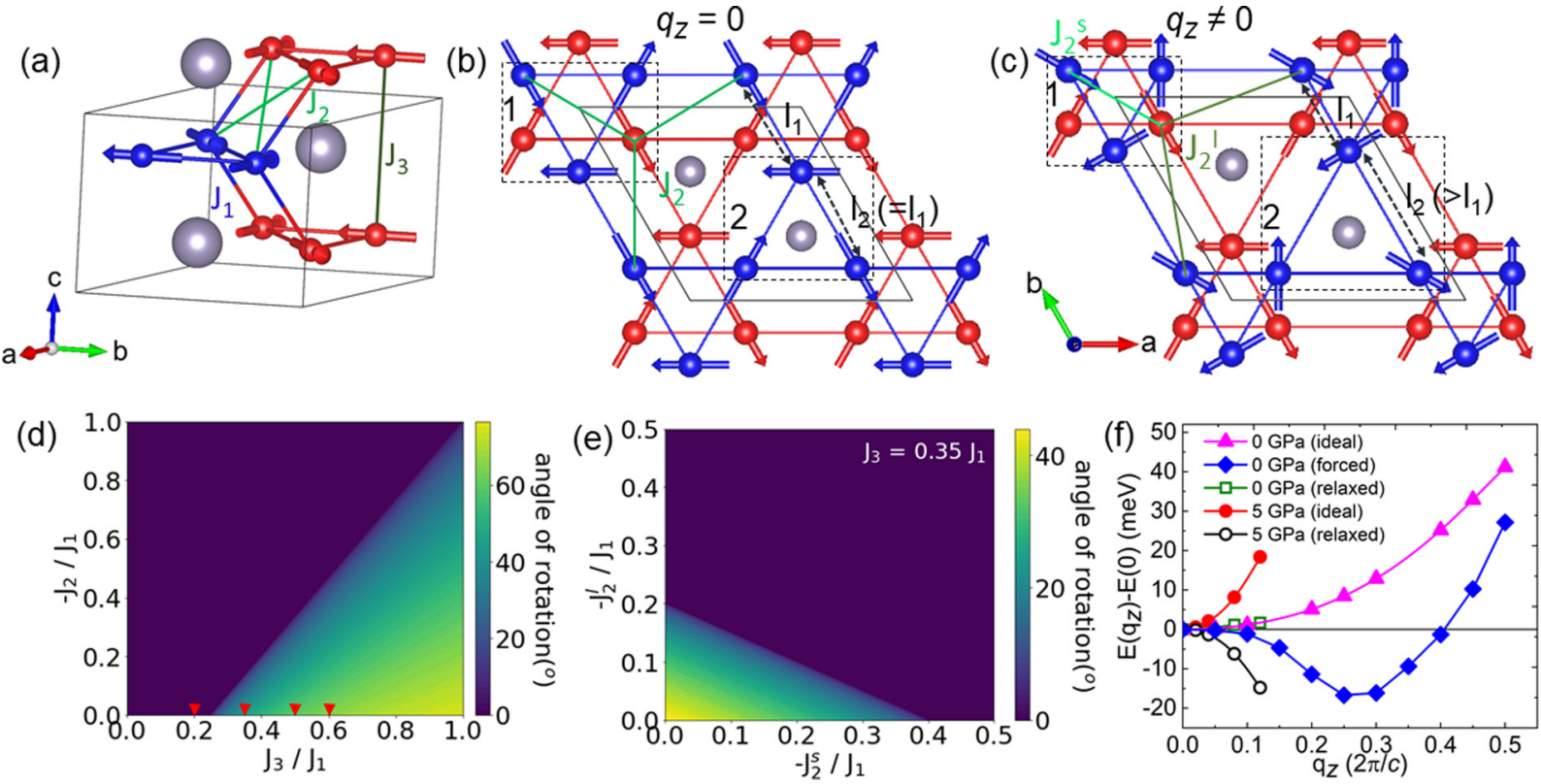

FIG. 1. (a) Unit cell for hexagonal $\mathrm{Mn}_{3} \mathrm{Sn}$ and stacking of three triangular layers (kagome layers) of Mn moments with $120^{\circ}$ AFM spin structure. Red and blue balls represent different layers of Mn atoms, whereas the $\mathrm{Sn}$ atoms are shown in gray balls. $J_{1}, J_{2}$, and $J_{3}$ represent first three out-of-plane (interlayer) neighboring exchange interactions between the Mn moments. (b) Top view of $\mathrm{Mn}_{3} \mathrm{Sn}$ where two ideal kagome sublattices of Mn atoms are stacked. The in-plane bond lengths $l_{1}$ and $l_{2}$ of the equilateral triangles shown in dashed boxes " 1 " and " 2 " and here $\delta=\frac{l_{2}-l_{1}}{l_{2}+l_{1}}=0$. (c) Top view of the distorted (relaxed) kagome sublattice stacking in $\mathrm{Mn}_{3} \mathrm{Sn}$ with two different in-plane bond lengths $\left(l_{2}>l_{1}\right)$ for the equilateral triangles as shown in dashed boxes " 1 " and " 2 " with $\delta \neq 0$. Splitting of $J_{2}$ to $J_{2}^{l}$ and $J_{2}^{s}$ is shown in green lines. (d) Phase diagram in the $J_{2}-J_{3}$ plane for ideal kagome layer stacking. $J_{2}$ and $J_{3}$ are measured in the unit of the magnitude of $J_{1}$. The color code indicates the angle of rotation of $\mathrm{Mn}$ triangle spins between two layers due to the helical modulation. The deep blue region indicates the $120^{\circ}$ triangular AFM state. (e) Phase diagram in the $J_{2}^{l}-J_{2}^{s}$ plane where the nonzero $\delta$ value results in splitting of $J_{2}$ to $J_{2}^{l}$ and $J_{2}^{s}$. The values marked by the down triangles on the $J_{3}$ axis in (d) are used for the phase diagrams shown in Fig. 8 (Appendix A). (f) Ab initio calculated spin spiral energy as a function of the spin spiral vector $\mathbf{q}=\left(0,0, q_{z}\right)$ where $120^{\circ}$ triangular AFM state corresponds to $\mathbf{q}=0$. Energies of the spin spiral state are calculated with respect to the $120^{\circ} \mathrm{AFM}$ state at zero pressure for $\delta=0$ (filled triangles), $\delta=2.4$ (open squares), and $\delta=12.0$ (filled diamonds). The data for $\delta=0$ at the zero pressure (filled triangles) are divided by a factor of ten for better visibility and comparison in the plot. The $\mathbf{q}$ dependence of the spiral energy at pressure $5 \mathrm{GPa}$ in the case of ideal $(\delta=0)$ and relaxed $(\delta=10.34)$ kagome structure is also shown with filled and open circles, respectively. The symbols are calculated data points and the solid lines are to guide the eye. The negative slope in the dispersion curves indicates the helical modulation of the $120^{\circ}$ triangular AFM state along $z$.

opposite chirality picks up an extra Berry phase, and consequently a large AHE has been observed in the case of $\mathrm{Mn}_{3} \mathrm{Sn}$ and $\mathrm{Mn}_{3} \mathrm{Ge}$. Since the topological Weyl nature, and hence the AHE of these materials, is critically inherited from the special kind of magnetic structure, precise control over the magnetic state is essential for any possible switching of the AHE.

$\mathrm{Mn}_{3} \mathrm{Sn}$ exhibits distinct AFM ground states in different temperature regimes depending upon the Mn concentration in the system [23]. While most of the samples show an in-plane triangular AFM ordering at room temperature, the presence of a slightly canted triangular AFM structure has also been found at very low temperatures $[24,25]$. The recent observation of large THE below $50 \mathrm{~K}$ in $\mathrm{Mn}_{3} \mathrm{Sn}$ corroborates the existence of noncoplanar magnetic structure at low temperatures [10]. While the samples used by Kuroda et al. [21] display an additional magnetic transition below $50 \mathrm{~K}$, the presence of a sharp transition around $240 \mathrm{~K}$ related to the helical magnetic ordering has also been observed in other systems [26]. Irrespective of the nature of the low temperature transitions, all the samples display a large AHE at room temperature. However, the AHE ceases to zero below $240 \mathrm{~K}$ when the system enters into a spin spiral (SS) state [26]. Using $a b$ initio calculations and Monte Carlo simulations, Park et al. have demonstrated the presence of an incommensurate helical modulation of the triangular spin structure that propagates along the $c$ direction [27]. Interestingly, no Weyl nodes were found in the electronic band structure for the SS magnetic state, explaining the absence of AHE in the helical magnetic phase [27]. Therefore, it is important to investigate the correlation between the two observed magnetic ground states of $\mathrm{Mn}_{3} \mathrm{Sn}$ to explore the possibility of controlling the transition between them. Motivated by the aforementioned discussion, herein, we utilize hydrostatic pressure as a control parameter to probe the switching mechanism of AHE in the case of the noncollinear antiferromagnetic $\mathrm{Mn}_{3} \mathrm{Sn}$.

\section{METHODS}

The density functional theory (DFT) calculations were employed to study the magnetic properties of the present system. The spin polarized calculations were carried out within the projector augmented wave (PAW) method [28] as implemented in the Vienna Ab initio Simulation Package (VASP) [29-32]. The generalized gradient approximation 
(GGA) was used as the exchange-correlation potential in the form of Perdew-Burke-Ernzerhof (PBE) [33]. For the Brillouin zone (BZ) integration, a $\Gamma$-centered $k$-point grid of $8 \times 8 \times 9$ was used with a plane-wave cutoff energy of $500 \mathrm{eV}$. Note that the noncollinear version of the code was utilized for determining the conventional magnetic ground state in this type of hexagonal system. The experimentally obtained lattice constants were kept fixed in our calculations where the internal coordinates were relaxed till the forces become less than $1 \mathrm{meV} / \AA$. Further, the spin spiral calculations were performed using the full potential linearized augmented plane wave (FLAPW) method implementing the noncollinear version of the FLEUR code [34]. The energy of the SS state characterized by wave vector $\mathbf{q}$ was calculated using the generalized Bloch theorem [35]. We have also employed the PBE exchange-correlation functional as the GGA approximation. We have considered a $16 \times 16 \times 18$ mesh for $k$ points in the full BZ and a cutoff of $K_{\max }=4.1 \mathrm{a.u}^{-1}$ to expand the LAPW basis functions. The $\mathbf{q}=0$ state was considered as a perfect $120^{\circ}$ triangular spin configuration on each kagome sublattice.

Polycrystalline ingots of $\mathrm{Mn}_{3+x} \mathrm{Sn}_{1-x}$ with $x=0.05,0.04$, and 0.03 were prepared by arc melting stoichiometric amounts of highly pure $\mathrm{Mn}$ and $\mathrm{Sn}$ metals under argon atmosphere. The samples were melted multiple times to ensure a better homogeneity. The as-prepared ingots were annealed for eight days in evacuated quartz tubes at a temperature of $1073 \mathrm{~K}$, followed by slow cooling to room temperature. Room temperature $\mathrm{x}$ ray powder diffraction measurements were performed using a Rigaku SmartLab x-ray diffractometer with a $\mathrm{Cu}-\mathrm{K} \alpha$ source. Low temperature $\mathrm{x}$-ray diffraction (XRD) measurements were carried out by utilizing a low temperature attachment (Oxford PheniX) to the X-ray diffractometer (PANalytical). To investigate the crystal structure with applied pressure, XRD measurements were performed using a diamond anvil cell at the beamline BL-11, INDUS-II synchrotron radiation source (Raja Ramanna Centre for Advanced Technology, Indore, India). Magnetic and transport measurements were carried out using a Quantum Design (QD) SQUID magnetometer and a QD physical property measurement system (PPMS), respectively. Pressure dependent magnetic and transport studies were conducted using respective pressure cells provided by Quantum Design.

\section{RESULTS AND DISCUSSION}

It is very important to understand the underlying magnetic states which govern the control and switching of AHE in $\mathrm{Mn}_{3} \mathrm{Sn}$. The primary contributions to the total energy of the magnetic state can be narrowed down to the exchange interactions, Dzyaloshinskii-Moriya interaction (DMI), and anisotropy energy. Since the inverse triangular spin structure in $\mathrm{Mn}_{3} \mathrm{Sn}$ is mainly stabilized by geometrical frustration, the role of DMI to influence the long-range magnetic order in the present centrosymmetric system is extremely limited [36], except breaking the degeneracy of the sense of rotation. The contribution from the anisotropy energy can also be safely ruled out for the helical modulation of an in-plane triangular spin structure along the $z$ axis. Thus, the competing out-ofplane exchange interactions primarily determine the helical modulation in the system. To understand the origin of the helically modulated magnetic ground state, we first analyze the role of different exchange parameters within the classical Heisenberg model, $H=\sum_{i>j} J_{i j} \hat{n}_{i} \cdot \hat{n}_{j}$, where the exchange constants $J_{i j}$ determine the strength and nature of interactions between Mn moments pointing along the unit vector $\hat{n}$.

In the hexagonal $\mathrm{Mn}_{3} \mathrm{Sn}$ structure each $\mathrm{Mn}$ atom in the kagome plane has four nearest neighbors, where two equilateral triangles are connected by a common $\mathrm{Mn}$ atom [see the lattice with red and blue Mn balls in Fig. 1(b)]. However, it has been reported that $\mathrm{Mn}_{3} \mathrm{Sn}$ forms a distorted kagome lattice where one of the Mn triangles shrinks while the other expands as shown in Fig. 1(c) [37]. Here, we first consider the case of an ideal kagome sublattice structure and try to find out the role of frustration in the exchange parameters in stabilizing the SS ground state. For this purpose, we made a reasonable assumption that the in-plane exchange constants do not play any role in stabilizing the helical order along the $z$ direction. Therefore, we fixed the in-plane magnetic structure to a perfect $120^{\circ}$ triangular AFM configuration. The first three interplane nearest-neighbor exchange parameters, $J_{1}, J_{2}$, and $J_{3}$ as labeled in Fig. 1(a), are taken into consideration in order to study the transition from noncollinear AFM state to helically modulated magnetic state. In the inelastic neutron scattering experiment, $J_{1}$ and $J_{3}$ were reported to be AFM in nature while $J_{2}$ was found to be ferromagnetic (FM) [27]. The energy of the magnetic state per Mn atom is now translated into the following classical exchange Hamiltonian,

$$
\begin{aligned}
H= & 2 J_{1} \cos \left(\frac{2 \pi}{3}+\theta\right)+2 J_{1} \cos \left(\frac{2 \pi}{3}-\theta\right) \\
& +6 J_{2} \cos \theta+2 J_{3} \cos (2 \theta) .
\end{aligned}
$$

Here, $\theta$ is the measure of helical modulation, i.e., the angle of rotation between two successive layers of spin triangles connected with the SS vector $\mathbf{q}$ and $\frac{2 \pi}{3}$ in $J_{1}$ is the initial phase between two consecutive kagome sublattices. The value of $\theta$ varies from 0 to $180^{\circ}$ and the corresponding lowest energy state is the ground state in the $J_{2}-J_{3}$ plane. Here, both $J_{2}(\leqslant 0)$ and $J_{3}(\geqslant 0)$ are relative quantities with respect to the magnitude of $J_{1}$. The results presented in Fig. 1(d) show a sharp boundary between the noncollinear AFM ordering with and without the helical modulation along $z$. We find that the competing $J_{2}$ and $J_{3}$ drive the system into the helical SS state, indicating that the exchange frustration is the key route for achieving the spiral modulation along $z$. It is important to mention here that the SS ground states stabilized by competing exchange interactions have also been found in hexagonal [38] and square [39] lattices.

The ideal kagome sublattice in $\mathrm{Mn}_{3} \mathrm{Sn}$ is expected to be distorted into two unequal equilateral triangles as discussed above. Therefore, we define an effective trimerization parameter, $\delta$, as $\frac{l_{2}-l_{1}}{l_{2}+l_{1}}$, where $l_{1}$ and $l_{2}$ are the bond lengths corresponding to two equilateral triangles formed by $\mathrm{Mn}$ atoms in the kagome sublattice [see dashed square " 1 " and " 2 " in Figs. 1(b) and 1(c)]. One can consider $\delta$ as the effective trimerization parameter where the in-plane exchange coupling within two unequal triangles can be changed significantly $[27,40]$. Here we note that $\delta=0$ defines the ideal kagome lattice. Since a nonzero value of $\delta$ causes bond length 
TABLE I. $\delta$, the measure of effective trimerization at different pressure values in the case of ideal and relaxed structures, along with Mn Wyckoff position.

\begin{tabular}{lcc}
\hline \hline Pressure $(\mathrm{GPa})$ & $x(6 \mathrm{c}: x, 2 x, 0.25)$ & $\delta\left(10^{-2}\right)$ \\
\hline 0.0 (ideal) & 0.1666 & 0.0 \\
5.23 (ideal) & 0.1666 & 0.0 \\
0.0 (forced) & 0.1466 & 12.0 \\
0.0 (relaxed) & 0.1626 & 2.4 \\
2.75 (relaxed) & 0.1605 & 3.7 \\
5.23 (relaxed) & 0.1493 & 10.34 \\
\hline \hline
\end{tabular}

mismatch related to the exchange constant $J_{2}$, the corresponding large and small out-of-plane exchange constants $J_{2}^{l}$ and $J_{2}^{s}$ are expected to change their strength significantly and even their sign too. We, therefore, extend our analyses by constructing phase diagrams in the $J_{2}^{l}-J_{2}^{s}$ plane for several values of $J_{3}$ marked by arrows in Fig. 1(d). Figure 1(e) shows the $J_{2}^{l}-J_{2}^{s}$ phase diagram for $J_{3}=0.35$. Similar phase diagrams for $J_{3}=$ 0.2 (inside the nonhelical AFM phase) and $J_{3}=0.6$ (inside the helical AFM phase) with all possible "sign" combinations of $J_{2}^{l}$ and $J_{2}^{s}$ are shown in Fig. 8 (Appendix A). It is evident that the area of the helical magnetic state increases with increasing $J_{3}$ value, i.e., the $\frac{J_{3}}{J_{1}}$ ratio. It is therefore remarkable that the control in $\delta$ values plays a crucial role in determining the magnetic state of the hexagonal systems like $\mathrm{Mn}_{3} \mathrm{Sn}$.

The conventional $120^{\circ}$ triangular AFM ground state can undergo a phase transition into the helical SS state by means of effective trimerization, which may bring significant changes in $J$ 's due to the changes in bond lengths. Hence, the effect of pressure $P$ in the hexagonal $\mathrm{Mn}_{3} \mathrm{Sn}$ system has been carried out within $a b$ initio electronic structure calculations where hydrostatic pressure $P$ is used as a parameter. The last column in Table I lists the $\delta$ values for various pressures. The corresponding Wyckoff position of the Mn atom at the middle column determines the kagome sublattice structures. Interestingly, the application of pressure, chemical as well as hydrostatic, results in a nonzero $\delta$ value that keeps on increasing with increasing pressure. Figure 1(f) summarizes the calculated energy corresponding to the noncollinear magnetic SS states characterized by q. In order to understand the role of pressure on $\delta$, and hence the magnetic ground state, we have taken the $120^{\circ}$ inverse triangular AFM state as the initial state corresponding to $\mathbf{q}=0$. Subsequently, the triangular spin state is helically modulated for $q_{z} \neq 0$, as can be seen by comparing the spin configurations in Figs. 1(b) and 1(c). The ideal kagome sublattice stacked $\mathrm{Mn}_{3} \mathrm{Sn}$ shows noncollinear $120^{\circ}$ inverse triangular AFM structure as the ground state up to a hydrostatic pressure $P=5.23 \mathrm{GPa}$. The SS dispersion does not show any significant changes like softening or hardening near the ground state [see Fig. 9 (Appendix A)]. However, the forced structure with $\delta=0.12$ at ambient pressure $(P=0)$ shows a SS ground state with a period of about $16.3 \AA$ A Such a helically modulated spin structure is indeed stabilized by the effective trimerization of the system which is fostered by pressure.

To interpret our calculations, we map the results onto a classical Heisenberg model which allows us to determine

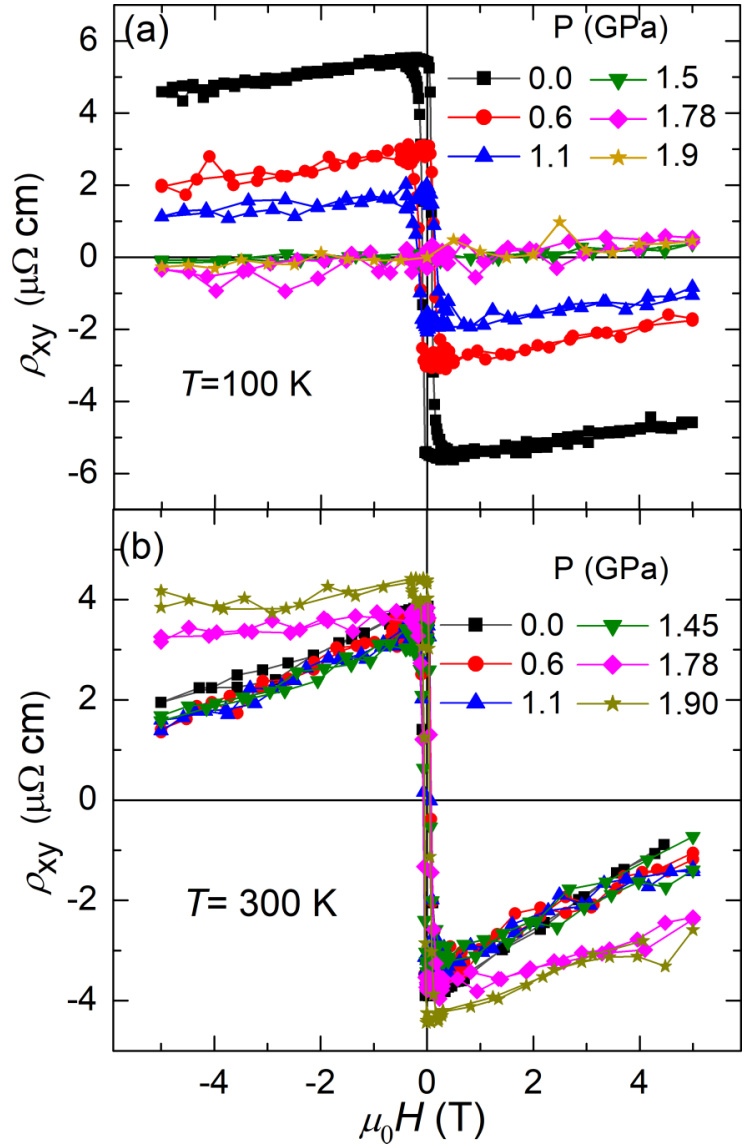

FIG. 2. Field dependence of Hall resistivity for $\mathrm{Mn}_{3.05} \mathrm{Sn}_{0.95}$ at different hydrostatic pressures measured at (a) $T=100 \mathrm{~K}$ and (b) $T=300 \mathrm{~K}$.

the first three or four inter-sublattice exchange constants as discussed earlier. A good fit is obtained by using $J_{1}, J_{2}$, and $J_{3}$ for the ambient pressure ideal structure where the nature of the exchange constants is similar to the experimental results [27]. In the case of forced structure at ambient pressure, the four exchange constants which include $J_{2}^{s}$ and $J_{2}^{l}$ give good fitting to the results with SS ground state. Details of the calculation are provided in Appendix A. We find that the ratio of $\frac{J_{3}}{J_{1}}$ does not change significantly when we move from the ideal to the forced trimerization case. Interestingly, $J_{2}^{s}$ changes sign to become antiferromagnetic while $J_{2}^{l}$ remains ferromagnetic. For the ambient pressure ideal structure, the relative values of $J_{2}$ and $J_{3}$ measured in the unit of $J_{1}$ are about 0.445 and 0.340 , respectively. The corresponding point lies in the dark blue region in Fig. 1(d). In the case of the forced trimer system, the $J_{3}$ value is about 0.336 , which is slightly smaller than the value used for constructing Fig. 1(e). This is indeed remarkable as our calculated $J_{2}^{s}$ and $J_{2}^{l}$ (in the unit of $J_{1}$ ) lie in the region of the SS phase in Fig. 1(e) [see Table II (Appendix A)]. Now, the relaxed structure at ambient pressure changes $\delta$ value from 0 (ideal) to 0.024 due to the chemical pressure effect. The SS energy dispersion calculated up to $\mathbf{q}=(0,0,0.125)$ shows significant softening, possibly due to the effective trimerization. The relaxed structure at $P=5.23$ GPa shows significantly large $\delta$ value of about 0.10 , which further establishes the role of pressure in stabilizing 


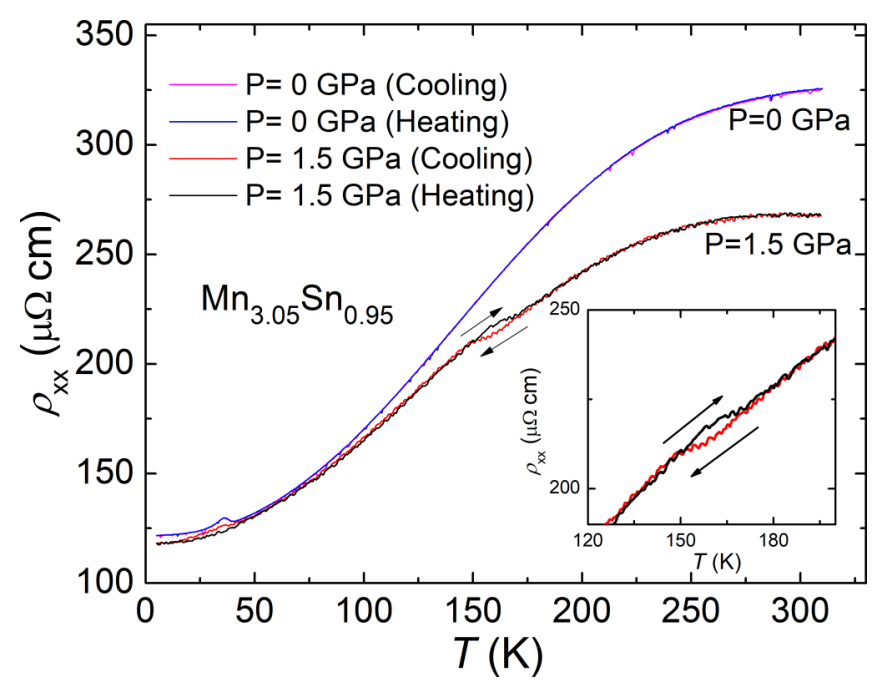

FIG. 3. Temperature dependent zero field longitudinal resistivity $\left[\rho_{x x}(T)\right]$ measured at ambient and 1.5 GPa pressure for $\mathrm{Mn}_{3.05} \mathrm{Sn}_{0.95}$. The measurement is performed both in cooling and heating modes as marked by arrows. The inset shows an amplified view of the $1.5 \mathrm{GPa}$ $\rho_{x x}(T)$ data around the phase transition region. A small kink at $35 \mathrm{~K}$ present in the zero pressure data corresponds to the instrumental artifact arising from the inconel used in the PPMS.

the helical ground state. Interestingly, the dispersion shows negative slope near $\mathbf{q}=0$, indicating the ground state at finite $\left(0,0, q_{z}\right)$ value. Hence, the pressure induced effective trimerization in the kagome sublattices modifies the frustrated exchange constants which ultimately determine the magnetic ground state.

Guided by our theoretical results, we set out to explore the possibility of controlling the magnetic ground state and its effect on the AHE in $\mathrm{Mn}_{3} \mathrm{Sn}$ with the help of hydrostatic pressure as a control parameter. The structural characterization for the samples studied in the present work can be found in Appendix B. Figure 2 shows the field dependence of anomalous Hall resistivity $\left(\rho_{x y}\right)$ measured at $100 \mathrm{~K}$ and $300 \mathrm{~K}$ at different applied pressures. As one can see, the $\rho_{x y}$ value at $100 \mathrm{~K}$ monotonically decreases from a large value of $5 \mu \Omega \mathrm{cm}$ at ambient pressure to almost zero by increasing the pressure to $1.5 \mathrm{GPa}$. It is worth mentioning that the magnitude of AHE at ambient pressure matches well with the previously reported value [4]. A further increase in pressure does not affect the anomalous component of $\rho_{x y}$ that remains at zero. Contrary to the $\rho_{x y}$ behavior at $100 \mathrm{~K}$, the anomalous Hall resistivity at $300 \mathrm{~K}$ remains almost constant when the pressure is changed from $0 \mathrm{GPa}$ to $1.45 \mathrm{GPa}$, before showing a slight increasing trend at higher pressures. To further support the observed pressure induced switching of AHE in the present system, we have measured temperature dependent longitudinal resistivity $\left[\rho_{x x}(T)\right]$ at ambient and $1.5 \mathrm{GPa}$ pressures (Fig. 3). The ambient pressure $\rho_{x x}(T)$ curves measured in both cooling and heating modes exhibit a metallic type of temperature dependency without the signature of any transition, as reported before [4]. In contrast, the $\rho_{x x}(T)$ data measured at 1.5 GPa display a transition-like anomaly with the appearance of a small hysteretic behavior between the cooling and heating $\rho_{x x}(T)$ curves at about $150 \mathrm{~K}$. The first-order nature of this
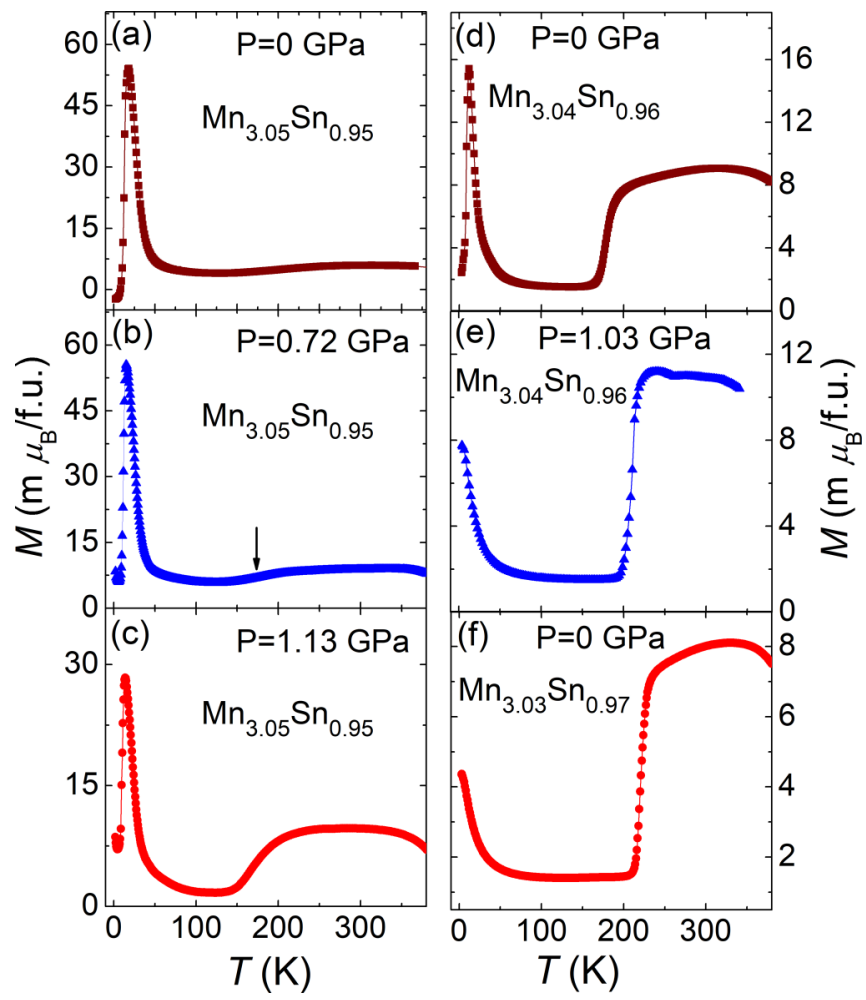

FIG. 4. Temperature dependence of zero field cooled (ZFC) magnetization $[M(T)]$ for $\mathrm{Mn}_{3.05} \mathrm{Sn}_{0.95}$ sample measured in an applied pressure of (a) $P=0 \mathrm{GPa}$, (b) $P=0.72 \mathrm{GPa}$, and (c) $P=$ $1.92 \mathrm{GPa} . M(T)$ curves for $\mathrm{Mn}_{3.04} \mathrm{Sn}_{0.96}$ sample measured in (d) $P=$ $0 \mathrm{GPa}$ and (e) $P=1.03 \mathrm{GPa}$. (f) $M(T)$ curve for $\mathrm{Mn}_{3.03} \mathrm{Sn}_{0.97}$ measured in ambient pressure. All the magnetization measurements were performed in an applied field of $0.1 \mathrm{~T}$.

transition suggests that the presence of a coupled magnetic and structural transition might be responsible for the pressure induced switching of AHE in the present system.

So far all the Hall effect measurements are carried out in the sample with chemical composition $\mathrm{Mn}_{3.05} \mathrm{Sn}_{0.95}$. To understand the contrasting nature of the pressure dependent AHE at $100 \mathrm{~K}$ and $300 \mathrm{~K}$, we have carried out a thorough magnetization study in the presence of hydrostatic pressure for three different samples with a small variation in the $\mathrm{Mn} / \mathrm{Sn}$ ratio. The temperature dependence of magnetization $[M(T)]$ curves measured at three different pressures and temperature up to $390 \mathrm{~K}$ are shown in Figs. 4(a)-4(c). The zero pressure $M(T)$ curve exhibits almost a temperature independent behavior down to $50 \mathrm{~K}$, below which the system undergoes a transition to the canted AFM state as reported earlier [25]. Since we mainly focus on the switching of AHE in the triangular AFM phase, the rest of our discussion will be concentrated on the results above $50 \mathrm{~K}$. The $M(T)$ curve measured at a pressure of $0.72 \mathrm{GPa}$ develops a small steplike feature at about $150 \mathrm{~K}$, marked by an arrow in Fig. 4(b). This additional transition at $150 \mathrm{~K}$ becomes very prominent when the pressure is further increased to $1.13 \mathrm{GPa}$ [see Fig. 4(c)]. To probe the origin of this new transition with pressure we have collected $M(T)$ data for $\mathrm{Mn}_{3.04} \mathrm{Sn}_{0.96}$ which consists of a slightly lower Mn concentration, as depicted in Fig. 4(d). Interestingly, the ambient pressure $M(T)$ data for $\mathrm{Mn}_{3.04} \mathrm{Sn}_{0.96}$ display a similar 


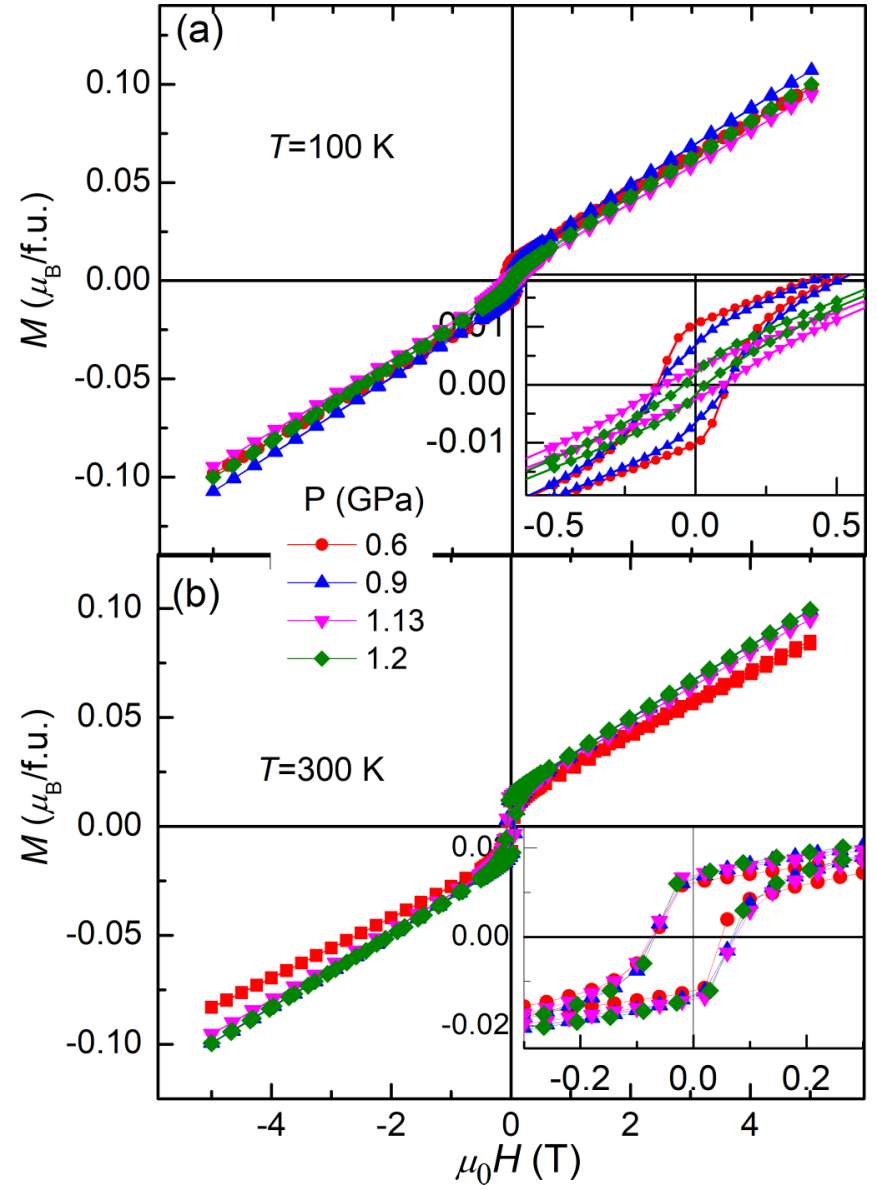

FIG. 5. Field dependent isothermal magnetization loops $[M(H)]$ for $\mathrm{Mn}_{3.05} \mathrm{Sn}_{0.95}$ sample measured at different hydrostatic pressures and at two temperatures (a) $T=100 \mathrm{~K}$ and (b) $T=300 \mathrm{~K}$. Insets: The magnified magnetization data in the low field regime.

transition to that is observed at $1.13 \mathrm{GPa}$ for $\mathrm{Mn}_{3.05} \mathrm{Sn}_{0.95}$. The additional transition in $\mathrm{Mn}_{3.04} \mathrm{Sn}_{0.96}$ shifts to about $200 \mathrm{~K}$ at $1.03 \mathrm{GPa}$ [Fig. 4(e)]. Similar pressure dependent shifting of the low temperature transition has also been reported earlier [41]. By further reducing the Mn concentration in $\mathrm{Mn}_{3.03} \mathrm{Sn}_{0.97}$, the ambient pressure transition in the $M(T)$ appears at around $220 \mathrm{~K}$ [Fig. 4(f)]. Here we note that like the $M(T)$ data, the ambient pressure $\rho_{x x}(T)$ data for $\mathrm{Mn}_{3.03} \mathrm{Sn}_{0.97}$ also display a transition around $220 \mathrm{~K}$ (see Appendix C). As mentioned in the introduction section, $\mathrm{Mn}_{3} \mathrm{Sn}$ exhibits multiple magnetic phases depending on the Mn concentration. The extra transition found in the case of $\mathrm{Mn}_{3.04} \mathrm{Sn}_{0.96}$ and $\mathrm{Mn}_{3.03} \mathrm{Sn}_{0.97}$ at ambient pressure corresponds to the magnetic phase transition from the high temperature triangular AFM state to the helical AFM phase at low temperatures. The application of hydrostatic pressure shifts this transition to higher temperatures. Hence the appearance of the extra transition in the case of $\mathrm{Mn}_{3.05} \mathrm{Sn}_{0.95}$ actually represents a pressure induced magnetic phase transition from the conventional triangular AFM to the helical AFM phase. Since the helical AFM structure does not support any AHE, the observation of switching of anomalous Hall resistivity in Fig. 2(a) indeed corresponds to the magnetic phase transition in the sample.
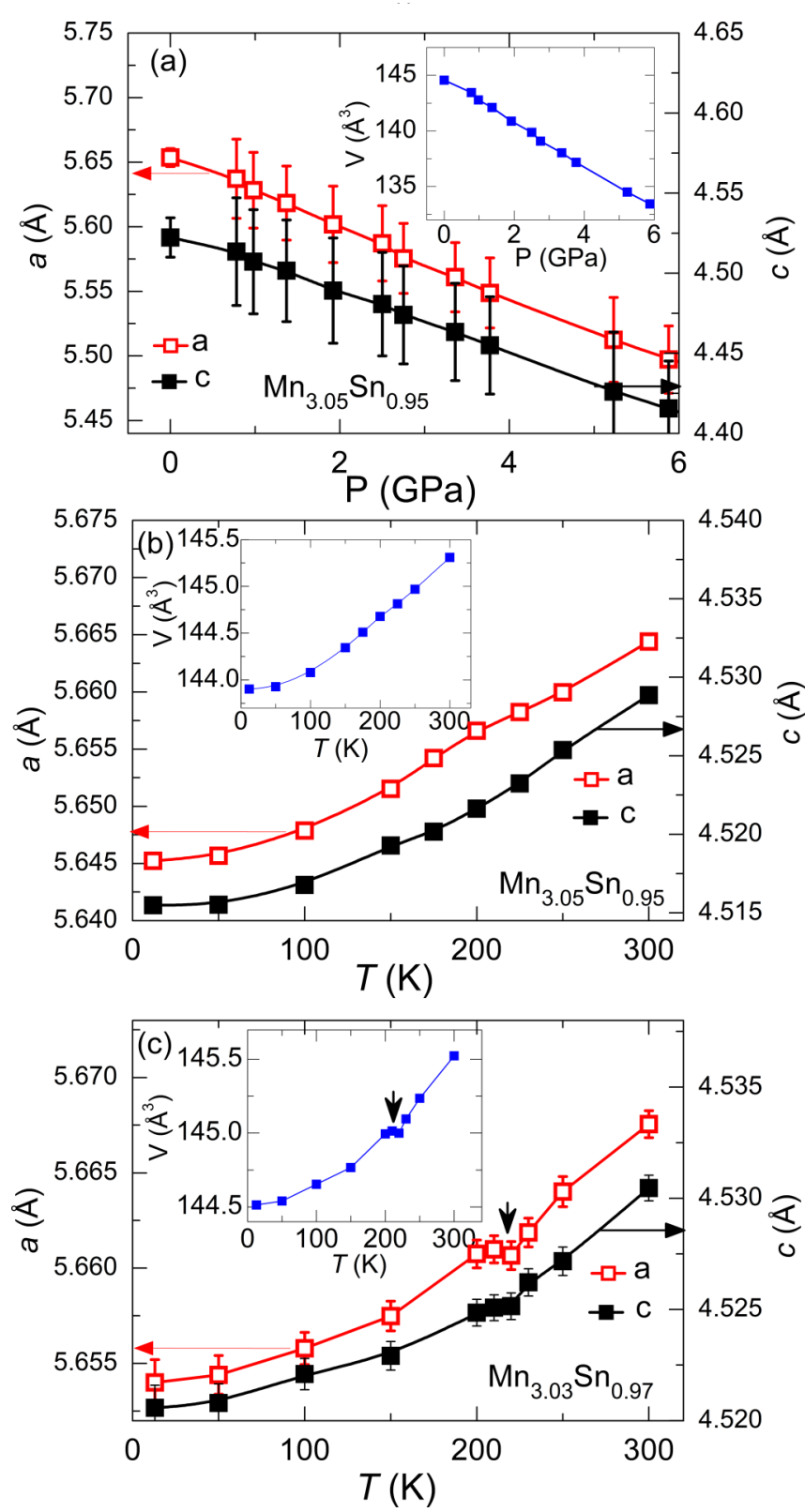

FIG. 6. (a) Pressure dependence of lattice parameters $a$ (left ordinate) and $c$ (right ordinate) for the hexagonal $\mathrm{Mn}_{3.05} \mathrm{Sn}_{0.95}$ calculated at room temperature. Inset: The unit cell volume as a function of hydrostatic pressure. Temperature dependence of lattice parameters $a$ and $c$ for (b) $\mathrm{Mn}_{3.05} \mathrm{Sn}_{0.95}$ and (c) $\mathrm{Mn}_{3.03} \mathrm{Sn}_{0.97}$ in ambient pressure. Insets of (b) and (c) present the unit cell volume as a function of temperature. The temperature at which the change of lattice parameters and unit cell volume occurs is marked by a downward arrow.

Our pressure dependent $M(T)$ measurements for different $\mathrm{Mn}_{3} \mathrm{Sn}$ samples provide an excellent correlation between the magnetic structures and the observed AHE. To further establish the connection, we have carried out isothermal magnetization measurements $[M(H)]$ at different pressures at $T=$ $100 \mathrm{~K}$ and $300 \mathrm{~K}$, as shown in Fig. 5. The $M(H)$ loops measured at $100 \mathrm{~K}$ exhibit a nearly linear behavior up to $5 \mathrm{~T}$ and remain almost unchanged with applied pressure up to $1.2 \mathrm{GPa}$ [Fig. 5(a)]. However, a close look at the low field regime [see 
the inset of Fig. 5(a)] reveals a unique pressure dependency of the magnetization. It is worth mentioning that the triangular AFM phase in $\mathrm{Mn}_{3}$ Sn displays a small spontaneous magnetization owing to a tiny deviation from the $120^{\circ}$ structure due to the alignment of $\mathrm{Mn}$ moments with their respective local easy axes [42]. In the present case, this residual spontaneous magnetization can even be seen for pressure of $0.6 \mathrm{GPa}$. When the system transforms to the helical AFM phase at higher pressure, the magnitude of the spontaneous magnetization starts decreasing. Finally, a nearly linear kind of $M(H)$ loop is obtained for $P=1.2 \mathrm{GPa}$. The disappearance of the residual spontaneous magnetization at higher pressures directly supports our previous finding of a pressure induced helical magnetic state, where the uncompensated in-plane magnetic vector sums up to zero when it is rotated by $360^{\circ}$ in a single helical pitch. In corroboration with the $100 \mathrm{~K}$ Hall resistivity data, no pressure variation in the spontaneous magnetization is found for the $M(H)$ loop measured at $300 \mathrm{~K}$, as depicted in Fig. 5(b). These findings thoroughly establish the correlation between the pressure induced switching of the AHE and magnetic ground states.

Finally, pressure dependent XRD measurement is carried out at room temperature to find out the role of pressure on the crystal structure. Figure 6(a) shows the variation of lattice parameters $a$ and $c$ with hydrostatic pressure for $\mathrm{Mn}_{3.05} \mathrm{Sn}_{0.95}$. Both $a$ and $c$ decrease linearly with increasing pressure without showing any signature of structural phase transition up to a pressure of $10 \mathrm{GPa}$ (see the XRD pattern in Appendix B). The unit cell volume $(V)$ also changes linearly with pressure [see the inset of Fig. 6(a)]. Therefore, we rule out any role of structural phase transition in the observed switching of AHE. We have also performed temperature dependent XRD study at ambient pressure for $\mathrm{Mn}_{3.05} \mathrm{Sn}_{0.95}$ to find out any structural anomaly at low temperatures [Fig. 6(b)]. Both $a$ and $c$ as well as $V$ [inset of Fig. 6(b)] show a monotonic decrease with decreasing temperature, ruling out any temperature dependence of structural phase transition. It is also important to find out the relation between the crystal and magnetic structure in the helical AFM phase. Since our XRD measurements under pressure are limited to room temperature, we have selected $\mathrm{Mn}_{3.03} \mathrm{Sn}_{0.97}$ for the temperature dependent XRD study as this sample exhibits an ambient pressure helical phase transition around $220 \mathrm{~K}$. The temperature variation of lattice parameters for $\mathrm{Mn}_{3.03} \mathrm{Sn}_{0.97}$ is shown in Fig. 6(c). Interestingly, the inplane lattice parameter $a$ and $V$ display a dip-type feature at the helical phase transition. It is to be noted here that our theoretical study emphasizes the role of pressure induced trimerization of $\mathrm{Mn}$ atoms, where the in-plane bond length between neighboring Mn triangles changes, in stabilizing the helical AFM phase. Hence, the observed anomaly in the temperature dependent $a$ and $V$ [inset of Fig. 6(c)] with almost no change in the out-of-plane lattice parameter $c$ corroborate our theoretical proposition.

Although hydrostatic pressure stabilizes the helical phase at low temperature for $\mathrm{Mn}_{3.05} \mathrm{Sn}_{0.95}$, a similar transition is also found at ambient pressure for $\mathrm{Mn}_{3.03} \mathrm{Sn}_{0.97}$ with a larger unit cell volume. Here we note that irrespective of its exact composition, $\mathrm{Mn}_{3} \mathrm{Sn}$ intrinsically exhibits some amount of trimerization of the $\mathrm{Mn}$ atoms even at ambient pressure [37], consistent with our theoretical calculations. The stability of the helical phase depends on the relative strength of $J_{3}$ in the presence of competing $J_{2}^{l}$ and $J_{2}^{s}$ exchange constants. In the ideal structure of $\mathrm{Mn}_{3} \mathrm{Sn}$, there are six nearest neighbors for the Mn-Mn exchange constants connected by $J_{2}$ [three above and three below; see Fig. 1(b)]. The trimerization process splits the $J_{2}$ into a group of four $J_{2}^{l}$ and two $J_{2}$ [see Fig. 1(c)]. Hence, an overall decrease in the strength of $J_{2}$ is expected upon trimerization. As we can see from the phase diagram presented in Fig. 8 (Appendix A), the $J_{3}$ value is very sensitive in stabilizing the helically modulated phase. It is also evident from Fig. 1(d) that an overall decrease in the strength of $J_{2}$ increases the helical phase region. Therefore, the helically modulated phase is a result of the subtle balance between $J_{3}$ and the splitting of $J_{2}$ due to the trimerization. The higher cell volume in the case of $\mathrm{Mn}_{3.03} \mathrm{Sn}_{0.97}$ may intrinsically give lower effective $J_{2}$ values in comparison to that of $\mathrm{Mn}_{3.05} \mathrm{Sn}_{0.95}$. In addition, a different concentration of $\mathrm{Mn}$ atoms in these two samples also modifies the exchange interactions in the system. Hence, the observed different pressure effects in $\mathrm{Mn}_{3.05} \mathrm{Sn}_{0.95}$ and $\mathrm{Mn}_{3.03} \mathrm{Sn}_{0.97}$ depend not only on the volume of the unit cell but also on the position of extra Mn atoms and the effective exchange interaction strength in the system. Therefore, both positive and negative pressure may cause the helical AFM transition.

The present study exemplifies the mechanism for the switching of AHE in the $\mathrm{Mn}_{3} \mathrm{Sn}$ system by establishing a route to control the modulation by means of pressure. As the exchange interaction between the Mn moments depends directly on the bond length, the switching of ground state by exchange parameter variation can be achieved by control over the lattice parameters by means of pressure and strain. Though chemical pressure can bring about the required change in the lattice parameters, it cannot be used for the switching purpose in device applications. Hydrostatic pressure, on the other hand, has been widely used to manipulate the lattice parameters and even to bring about a structural transition in a system. The present mechanism to manipulate AHE differs completely from the recent reports of AHE switching in antiferromagnets [43-45]. Switching of AHE in thin films of $\mathrm{Mn}_{3} \mathrm{Pt}$ relies on the structural distortion controlled through electrical response in a piezoelectrical substrate, whereas in the case of bulk $\mathrm{Mn}_{3} \mathrm{Ge}$, the underlying mechanism for the pressure induced sign change of AHE is still unknown. In the present work, we have uncovered the process of trimerization in $\mathrm{Mn}_{3} \mathrm{Sn}$ that was not taken into consideration till now. In such a scenario, three nearest-neighbor Mn moments collectively respond to any external stimuli and in turn can greatly affect the magnetic properties at higher trimerization levels $[40,46]$. Now that we understand the ingredients for the stabilization of different ground states, the effect of Mn doping can also be explored to manipulate exchange parameters that will help to achieve switching at room temperature.

\section{CONCLUSION}

In conclusion, we have successfully demonstrated the switching of anomalous Hall effect in antiferromagnetic $\mathrm{Mn}_{3} \mathrm{Sn}$ using hydrostatic pressure. The theoretical analyses presented in this work establish a first hand link between different magnetic ground states of $\mathrm{Mn}_{3} \mathrm{Sn}$. Our experimental 
TABLE II. Out-of-plane exchange parameters as extracted by fitting the $E(\mathbf{q})$ vs $q$ curves with the usual exchange Hamiltonian.

\begin{tabular}{lcccc}
\hline \hline & & \multicolumn{2}{c}{$J_{2}(\mathrm{meV})\left[-J_{2} / J_{1}\right]$} & \\
\cline { 2 - 4 } & $J_{1}(\mathrm{meV})$ & $J_{2}^{l}(\mathrm{meV})\left[-J_{2}^{l} / J_{1}\right]$ & $J_{2}^{s}(\mathrm{meV})\left[-J_{2}^{s} / J_{1}\right]$ & $J_{3}(\mathrm{meV})\left[J_{3} / J_{1}\right]$ \\
\hline $0 \mathrm{GPa}$ (ideal) & 20.83 & $-0.32[0.024]$ & $-9.29[0.445]$ & \\
$0 \mathrm{GPa}$ (forced) & 13.12 & $-0.59[-0.197]$ & $4.409[0.340]$ \\
\hline \hline
\end{tabular}

results convincingly manifest the role of helical modulation of the inverse triangular spin structure to manipulate the AHE in the system. The pressure induced effective trimerization of the $\mathrm{Mn}$ atoms controls the exchange frustration which finally leads to the helical modulation. The present work could immensely help in the greater understanding of the extensive range of properties demonstrated by $\mathrm{Mn}_{3} \mathrm{Sn}$ and related materials.

\section{ACKNOWLEDGMENTS}

A. K. Nayak acknowledges the support from Department of Atomic Energy (DAE), Department of Science and Technology (DST) Ramanujan research grant (Grant No. SB/S2/RJN-081/2016), SERB research grant (Grant No. ECR/2017/000854), and Nanomission research grant [Grant No. SR/NM/NS-1036/2017(G)] of the Government of India. A. K. Nandy acknowledges the support of DAE and SERB research grant (Grant No. SRG/2019/000867) of the Government of India. A. K. Nandy thanks Prof. P. M. Oppeneer for the Swedish National Infrastructure for Computing (SNIC) facility and Dr. Gustav Bihlmayer for the computational support, JURECA at JSC, Forschungszentrum, Jülich, Germany. V.S. and R.N. acknowledge BRNS, India, for financial support bearing sanction Grant No. 37(3)/14/26/2017.

\section{APPENDIX A: THEORETICAL MODEL}

To understand the role of competing exchange interaction in stabilizing the helical modulation, we write down the classical Heisenberg Hamiltonian for a single $\mathrm{Mn}$ atom considering the inter-kagome sublattice coupling $J_{1}, J_{2}$, and $J_{3}$ [see Eq. (1)]. As $J_{1}$ is the strongest among three exchange parameters [27], we varied $J_{2}$ and $J_{3}$ in the units of $J_{1}$ over a range to obtain the ground state for all combinations. For every particular combination, we change the value of the angle of rotation $(\theta)$ between two layers from 0 to 90 degrees in order to determine the lowest energy state. After performing such calculations, two prototypical plots are shown in Fig. 7. The value of $\theta$ corresponding to the energy minima for these plots is assigned as the magnetic ground state. Here, Fig. 7(a) represents an unmodulated inverse triangular ground state while Fig. 7(b) shows the spin spiral (SS) energy for a helically modulated ground state. Similar calculations were performed for all the combinations and the corresponding phase diagrams are constructed.

In the case of trimerization with unequal equilateral triangles in the kagome sublattices, the bond corresponding to $J_{2}$ now splits into two unequal lengths. The Hamiltonian for this case is written as

$$
\begin{aligned}
H= & 2 J_{1} \cos \left(\frac{2 \pi}{3}+\theta\right)+2 J_{1} \cos \left(\frac{2 \pi}{3}-\theta\right) \\
& +2 J_{2}^{l} \cos (0+\theta)+2 J_{2}^{l} \cos (0-\theta)+J_{2}^{s} \cos (0+\theta) \\
& +J_{2}^{s} \cos (0-\theta)+J_{3} \cos (0+2 \theta)+J_{3} \cos (0-2 \theta),
\end{aligned}
$$

where $J_{2}^{l}\left(J_{2}^{s}\right)$ is the exchange constant corresponding to the larger (smaller) bond length. Similarly to the previous calculations, here, we have performed the energy calculations for all sign and strength combinations of $J_{2}^{l}$ and $J_{2}^{s}$, where $J_{1}$ and $J_{3}$ are fixed at constant values. The main text includes the phase diagram in the $J_{2}-J_{3}$ plane measured in the unit of $J_{1}$. In the case of effective trimerization, Fig. 8 contains the phase diagrams for various relative values of $J_{3}$ ranging from $J_{3} / J_{1}=0.2$ to 0.6 . From Fig. 8 , we conclude that the helical ground state can be achieved for various relative values of $J_{3}$. Importantly, the area of the SS phase in the $J_{2}^{l}-J_{2}^{s}$ plane is increasing with the relative strength of $J_{3}$. Here, we mention that these values of $J_{3}$ are from different regimes of the phase diagram calculated for an ideal kagome structure [marked in Fig. 1(d)]. $J_{3}=0.2,0.35,0.6$ are taken outside the helical
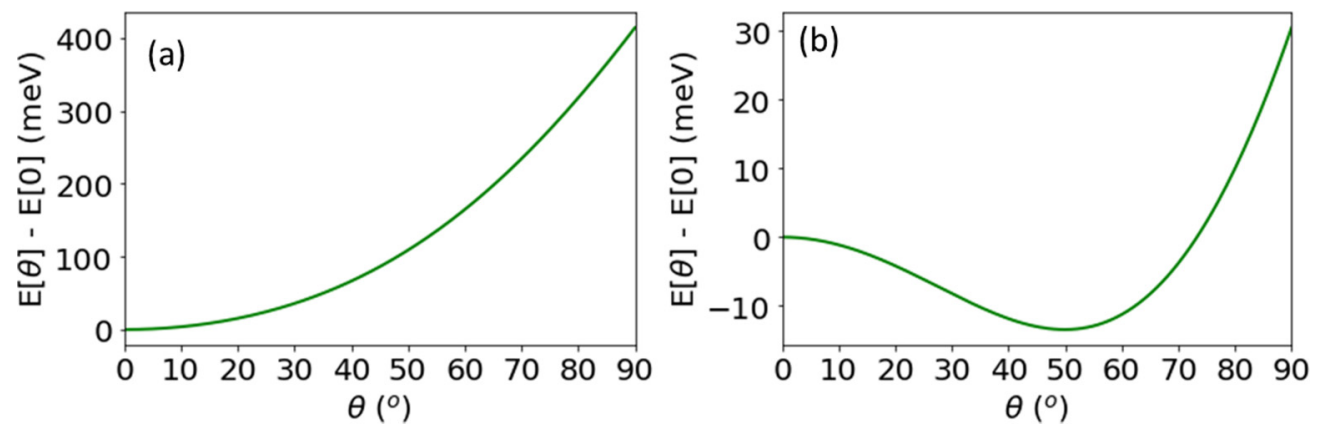

FIG. 7. Two prototype plots as obtained for (a) exchange parameters supporting a nonmodulated structure and (b) exchange parameters supporting a modulated structure. 

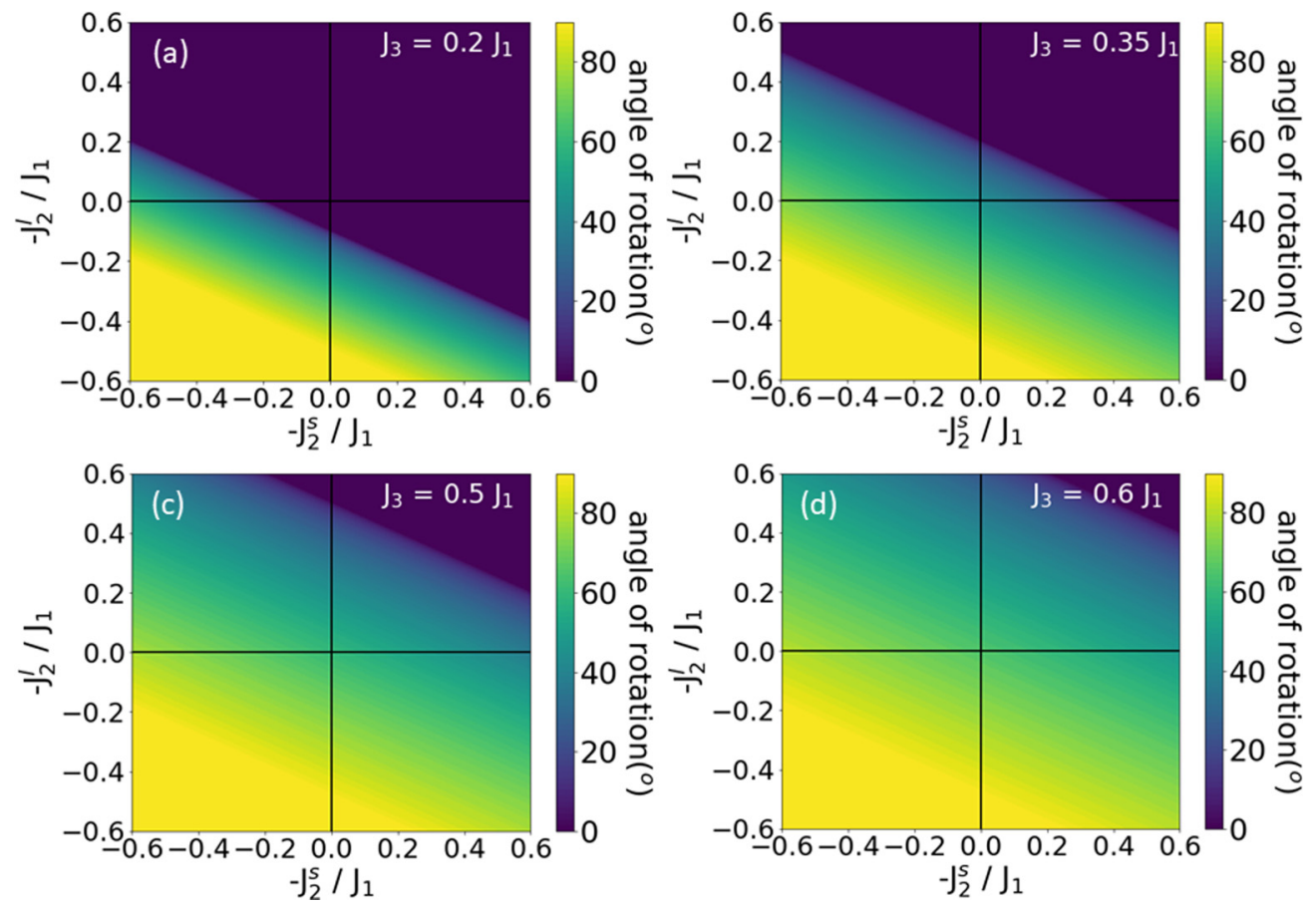

FIG. 8. Phase diagrams for the angle of rotation between two adjacent kagome layers in the $J_{2}^{s}-J_{2}^{l}$ plane with $J_{3}$ fixed at (a) $0.2 J_{1}$, (b) $0.35 J_{1}$, (c) $0.5 J_{1}$, and (d) $0.6 J_{1}$.

phase boundary, close to the phase boundary, and deep inside the helical phase, respectively. We also note that the sign change of either $J_{2}^{l}$ or $J_{2}^{s}$ favors the helically modified ground state, supporting our exchange constant values extracted from the DFT calculations. An increase in $J_{3}$ moves the phase boundary between zero and finite helical modulation toward higher $J_{2}$ and vice versa (Fig. 8).

\section{Spin spiral calculations}

The SS calculations were performed for different levels of trimerization due to nonzero pressure in the system. Figure 9 (a) shows the energy as a function of spin spiral vector $\mathbf{q}=\left(00 q_{z}\right)$ plot close to $\mathbf{q}=0$ (the main text includes the plot for the full range of $\mathbf{q}$ ). Note that the above $\theta$ value can be translated to $q_{z}$ in the SS calculations. From Fig. 9(a), we clearly see that in the case of ideal kagome sublattice stacking, the magnetic state does not show any helical modulation even at high pressure till $5 \mathrm{GPa}$. After structure relaxation, the energy curve at ambient pressure gets softer than that for ideal structure, but $\mathbf{q}=0$ still remains the magnetic ground state. However, the softening of the dispersion displays the importance of effective trimerization which is originating from the size mismatch of $\mathrm{Mn}$ and $\mathrm{Sn}$ atoms, i.e., the chemical pressure effect. Further increase in the trimerization at ambient pressure $(\delta=0.12)$ leads to a helical ground state corresponding to the $q_{z} \neq 0$ state. For the relaxed structure at $5 \mathrm{GPa}$ pressure, where the effect of trimerization is higher $(\delta=0.103)$ than that at ambient pressure $(\delta=0.024)$, the energy curve shows a large negative slope. This establishes a helically modulated SS to be the ground state with applying hydrostatic pressure as our experimental observations suggest. The SS curves were fitted using the Hamiltonian as in Eq. (1) for the ideal case, and Eq. (A1) for the trimerized case. Fitted profiles are shown
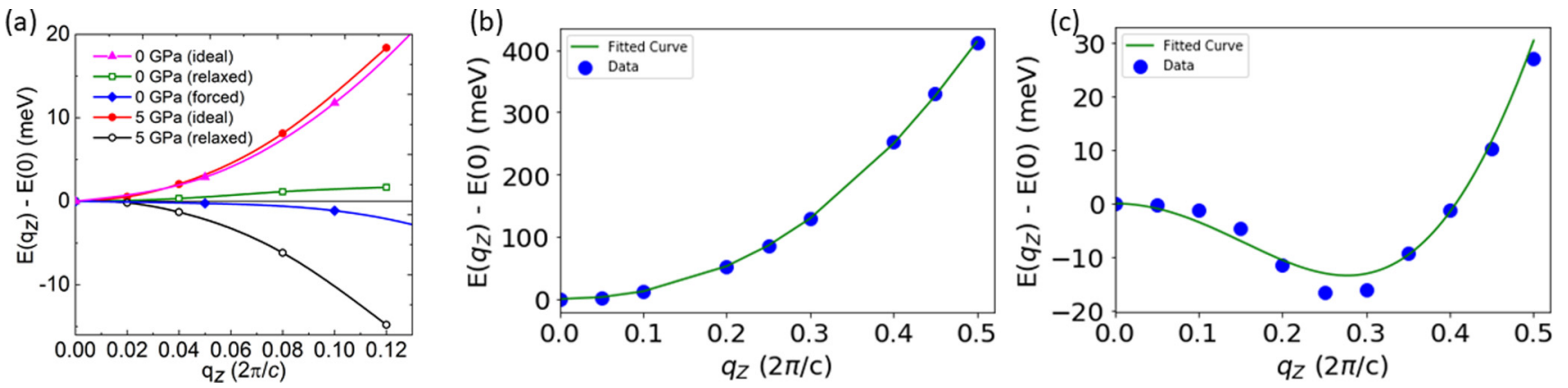

FIG. 9. (a) Spin spiral energy $E(\mathbf{q})$ vs $\mathbf{q}\left(00 q_{z}\right)$ as calculated for various pressure values with and without effective trimerization. $E\left(q_{z}\right)$ vs $q_{z}$ data points along with fitted curves for (b) ideal and (c) forced trimerized kagome sublattice at ambient pressure. 

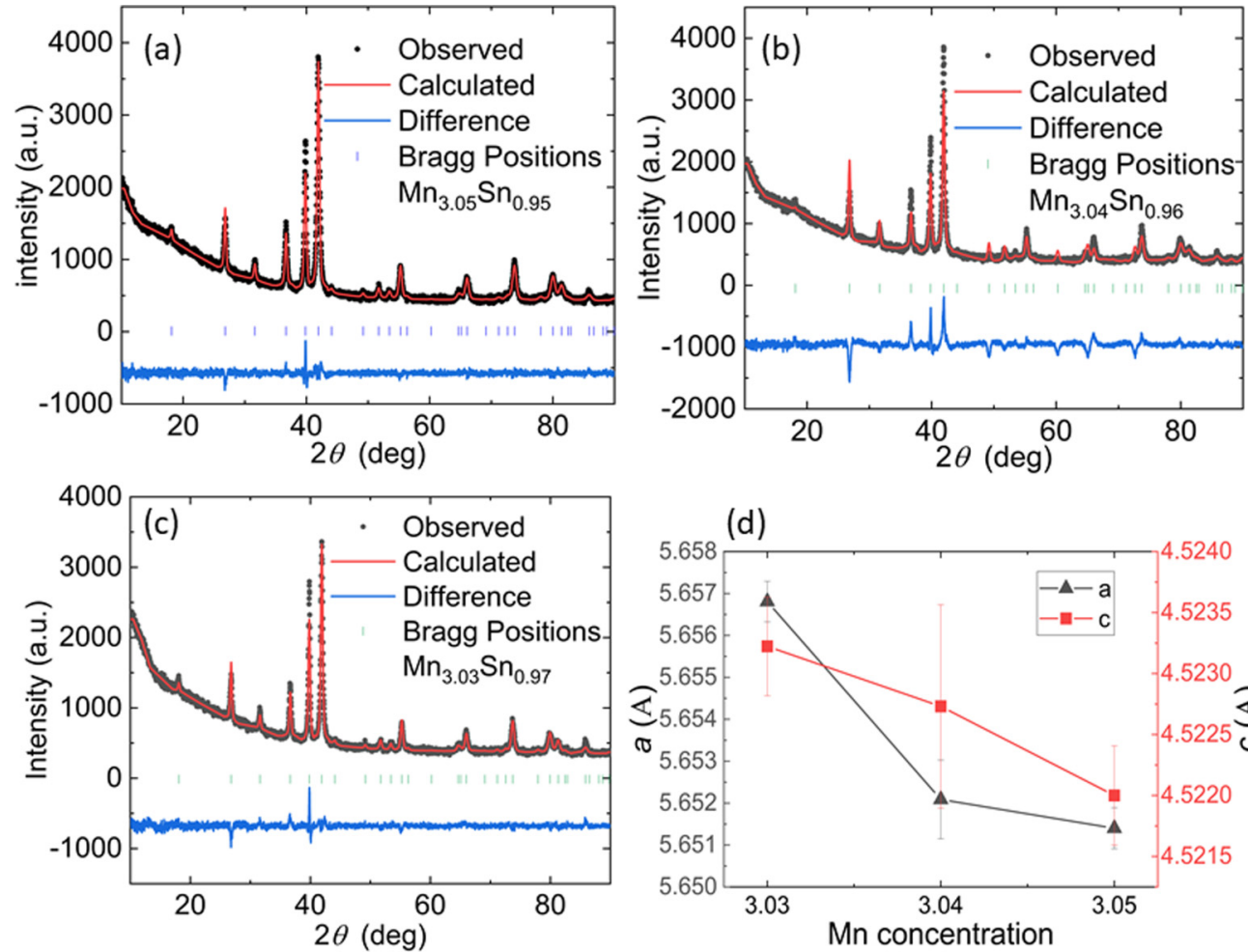

(d)

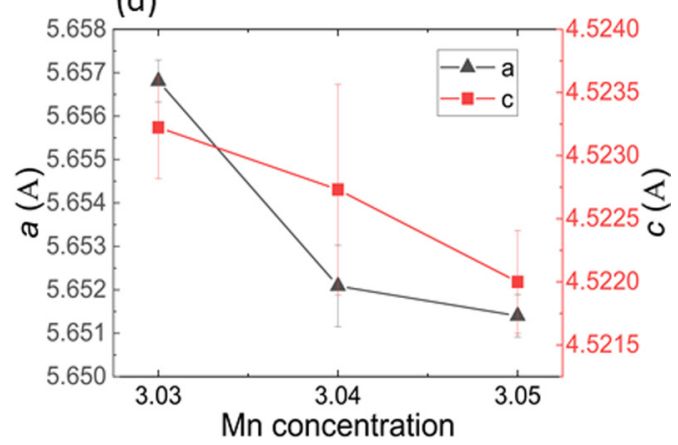

FIG. 10. XRD pattern with Rietveld refinement for (a) $\mathrm{Mn}_{3.05} \mathrm{Sn}_{0.95}$, (b) $\mathrm{Mn}_{3.04} \mathrm{Sn}_{0.96}$, and (c) $\mathrm{Mn}_{3.03} \mathrm{Sn}_{0.97}$ sample. (d) Calculated $a$ and $c$ values from the fitting.

in Figs. 9(b) and 9(c). The fitted exchange parameters are tabulated in Table II.

\section{APPENDIX B: X-RAY DIFFRACTION MEASUREMENTS}

XRD measurements were performed on polycrystalline samples using a Rigaku X-ray diffractometer. Figures 10(a)10 (c) show the fitted profile for different compositions of $\mathrm{Mn}_{3+x} \mathrm{Sn}_{1-x}$ samples. Fittings were performed taking into care the extra Mn concentration. Occupancy of respective sites was modified according to extra Mn. No extra impurity peaks were observed for any sample. Refined lattice parameters are plotted in Fig. 10(d). We find a small monotonic change in the lattice parameters with Mn composition.

\section{Pressure dependent XRD measurements}

Pressure dependence of XRD measurements was performed using the ECXRD (BL-11) beamline at the synchrotron radiation source beam facility at RRCAT, Indore, India. Figure 11(a) shows the ambient pressure XRD pattern with fitted profile for a $\mathrm{Mn}_{3.05} \mathrm{Sn}_{0.95}$ sample. The XRD pattern performed at such high fluence again establishes the single phase nature of the sample. Figures 11(b) and 11(c) show the fitted profile at finite pressure values. The presence of some additional peaks marked inside the figure originate from the high pressure gasket material used in the diamond anvil cell. We find that with the pressure cell in place, the intensity mismatch between the experimental and simulated patterns increases for some reflections. This may be due to some preferred orientation of grains inside the pressure cell.
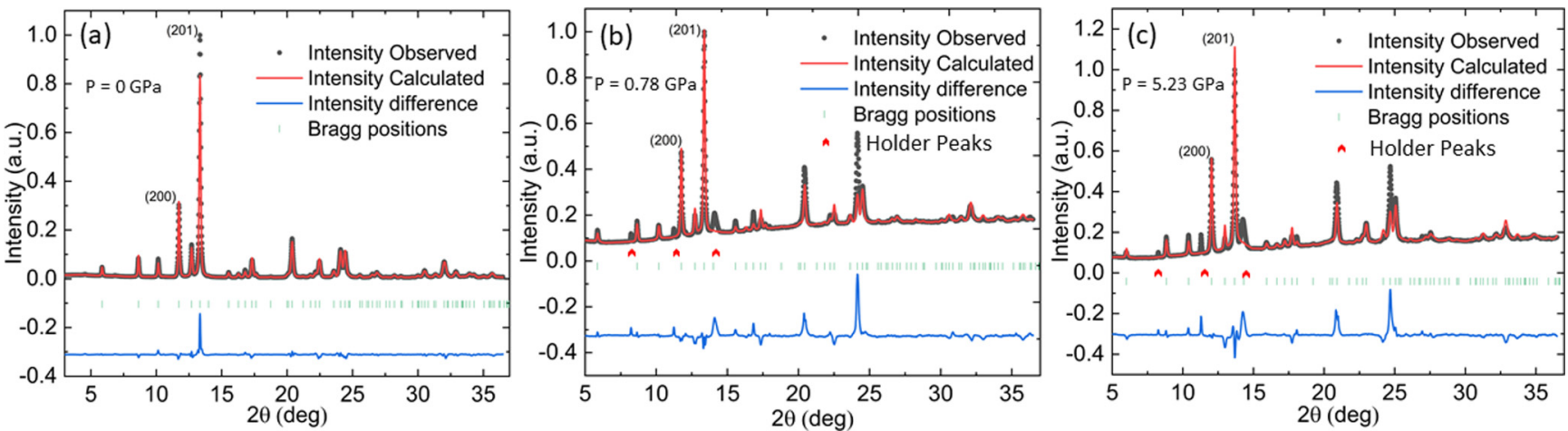

FIG. 11. XRD patterns with Rietveld refinement measured at (a) $0 \mathrm{GPa}$, (b) $0.78 \mathrm{GPa}$, and (c) $5.23 \mathrm{GPa}$. 

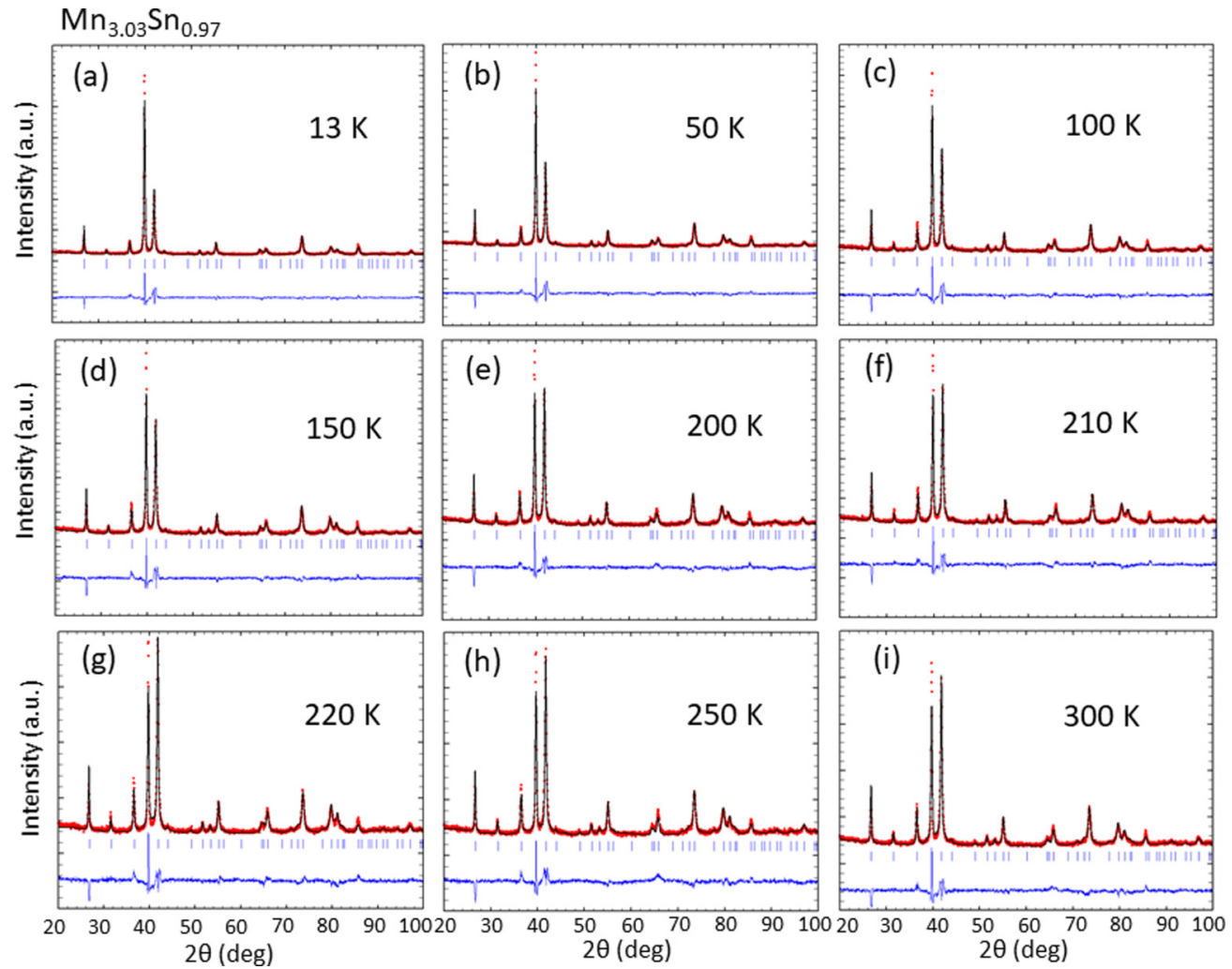

FIG. 12. Profile fitting graphs of XRD for $\mathrm{Mn}_{3.03} \mathrm{Sn}_{0.97}$ sample at various temperatures. Red circles represent the experimental data. Black line shows the calculated fitting. Difference between calculated and experimental graphs is shown as blue line. Straight blue ticks mark various Bragg positions.
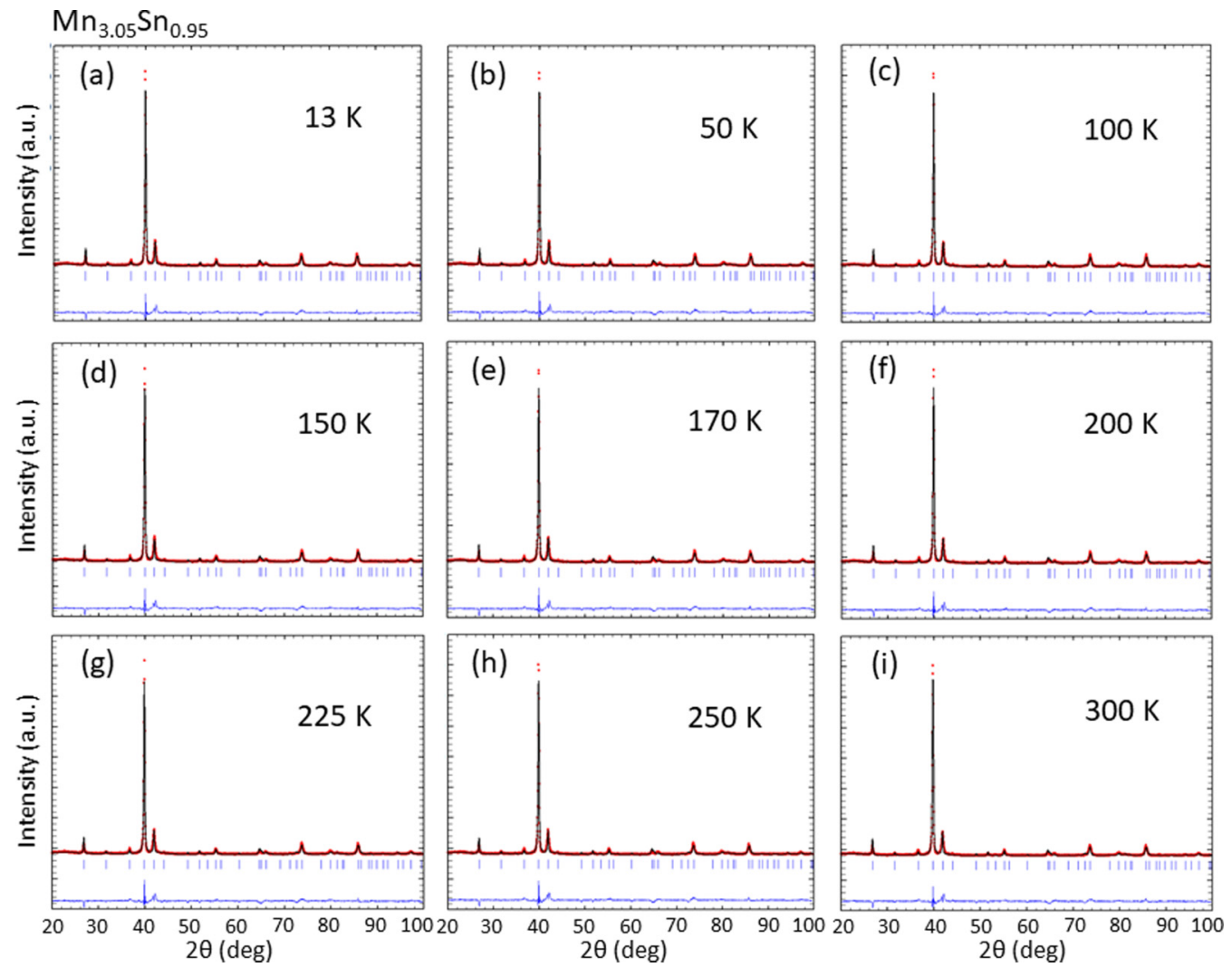

FIG. 13. Profile fitting graphs of XRD for $\mathrm{Mn}_{3.05} \mathrm{Sn}_{0.95}$ sample at various temperatures. Red circles represent the experimental data. Black line shows the calculated fitting. Difference between calculated and experimental graphs is shown as blue line. Straight blue ticks mark various Bragg positions. 


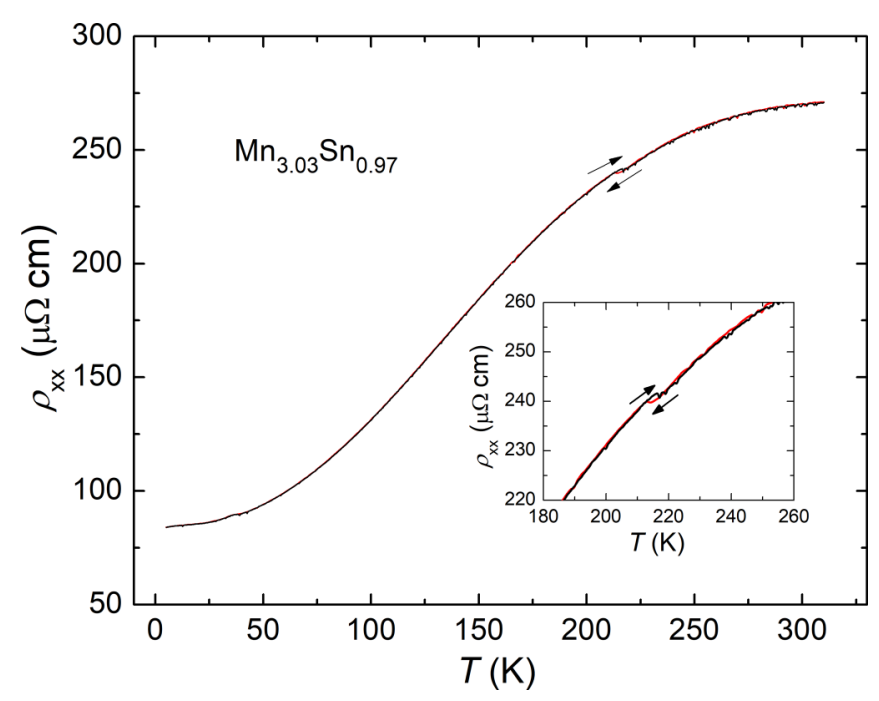

FIG. 14. Temperature dependence of resistivity measured at ambient pressure for $\mathrm{Mn}_{3.03} \mathrm{Sn}_{0.97}$. The inset shows the amplified view of the resistivity data around $200 \mathrm{~K}$. The presence of hysteresis between the cooling and heating resistivity data signifies the presence of a first-order phase transition.
This mismatch also explains the presence of larger error bars in lattice parameters obtained in the presence of pressure in the main text.

\section{Low temperature XRD measurements}

Low temperature XRD measurements were performed using a low temperature (Oxford PheniX) attachment to the $\mathrm{X}$-ray diffractometer (PANalytical). Figures 12 and 13 show the XRD patterns with fitting at different temperatures. No structural transition is visible down to $13 \mathrm{~K}$ in both the samples. Lattice parameters were first refined for room temperature XRD. Then, the refined parameters were used as a starting point for the refinement of the subsequent lower temperature. A similar process was followed for all the temperatures. Fitted lattice constants $a$ and $c$ are presented in the main text.

\section{APPENDIX C: RESISTIVITY MEASUREMENTS}

The temperature dependence of resistivity measured at ambient pressure for $\mathrm{Mn}_{3.03} \mathrm{Sn}_{0.97}$ is shown in Fig. 14.
[1] E. M. Pugh and N. Rostoker, Hall effect in ferromagnetic materials, Rev. Mod. Phys. 25, 151 (1953).

[2] N. Nagaosa, J. Sinova, S. Onoda, A. H. MacDonald, and N. P. Ong, Anomalous Hall effect, Rev. Mod. Phys. 82, 1539 (2010).

[3] H. Chen, Q. Niu, and A. H. MacDonald, Anomalous Hall Effect Arising from Noncollinear Antiferromagnetism, Phys. Rev. Lett. 112, 017205 (2014).

[4] S. Nakatsuji, N. Kiyohara, and T. Higo, Large anomalous Hall effect in a non-collinear antiferromagnet at room temperature, Nature 527, 212 (2015).

[5] A. K. Nayak, J. E. Fischer, Y. Sun, B. Yan, J. Karel, A. C. Komarek, C. Shekhar, N. Kumar, W. Schnelle, J. Kuebler, C. Felser, and S. S. P. Parkin, Large anomalous Hall effect driven by a nonvanishing Berry curvature in the noncollinear antiferromagnet $\mathrm{Mn}_{3} \mathrm{Ge}$, Sci. Adv. 2, e1501870 (2016).

[6] A. Mook, J. Henk, and I. Mertig, Thermal Hall effect in noncollinear coplanar insulating antiferromagnets, Phys. Rev. B 99, 014427 (2019).

[7] W. Feng, G.-Y. Guo, J. Zhou, Y. Yao, and Q. Niu, Large magneto-optical Kerr effect in noncollinear antiferromagnets $\mathrm{Mn}_{3} X(X=\mathrm{Rh}, \mathrm{Ir}, \mathrm{Pt})$, Phys. Rev. B 92, 144426 (2015).

[8] P. Němec, M. Fiebig, T. Kampfrath, and A. V. Kimel, Antiferromagnetic opto-spintronics, Nat. Phys. 14, 229 (2018).

[9] F. Bartram, S. Sorn, Z. Li, K. Hwangbo, S. Shen, F. Frontini, L. He, P. Yu, A. Paramekanti, and L. Yang, Anomalous Kerr effect in $\mathrm{SrRuO}_{3}$ thin films, Phys. Rev. B 102, 140408(R) (2020).

[10] P. K. Rout, P. V. Prakash Madduri, S. K. Manna, and A. K. Nayak, Field-induced topological Hall effect in the noncoplanar triangular antiferromagnetic geometry of $\mathrm{Mn}_{3} \mathrm{Sn}$, Phys. Rev. B 99, 094430 (2019).

[11] P. Wadley, B. Howells, J. Elezny, C. Andrews, V. Hills, R. Campion, V. Novak, K. Olejnik, F. Maccherozzi, S. Dhesi, S. Martin, T. Wagner, J. Wunderlich, F. Freimuth, Y. Mokrousov, J. Kune, J. Chauhan, M. Grzybowski, A. Rushforth, K.
Edmonds, B. Gallagher, and T. Jungwirth, Electrical switching of an antiferromagnet, Science 351, 587 (2016).

[12] L. Salemi, M. Berritta, A. K. Nandy, and P. M. Oppeneer, Orbitally dominated Rashba-Edelstein effect in noncentrosymmetric antiferromagnets, Nat. Commun. 10, 5381 (2019).

[13] T. Matsuda, N. Kanda, T. Higo, N. P. Armitage, S. Nakatsuji, R. Matsunaga, Room-temperature terahertz anomalous Hall effect in Weyl antiferromagnet $\mathrm{Mn}_{3} \mathrm{Sn}$ thin films, Nat. Commun. 11, 909 (2020).

[14] M. Kimata, H. Chen, K. Kondou, S. Sugimoto, P. K. Muduli, M. Ikhlas, Y. Omori, T. Tomita, A. H. MacDonald, S. Nakatsuji, and Y. Otani, Magnetic and magnetic inverse spin Hall effects in a non-collinear antiferromagnet, Nature (London) 565, 627 (2019).

[15] T. Higo, D. Qu, Y. Li, C. L. Chien, Y. Otani, and S. Nakatsuji, Anomalous Hall effect in thin films of the Weyl antiferromagnet $\mathrm{Mn}_{3} \mathrm{Sn}$, Appl. Phys. Lett. 113, 202402 (2018).

[16] T. Ikeda, M. Tsunoda, M. Oogane, S. Oh, T. Morita and Y. Ando, Anomalous Hall effect in polycrystalline $\mathrm{Mn}_{3} \mathrm{Sn}$ thin films, Appl. Phys. Lett. 113, 222405 (2018).

[17] J. Taylor, A. Markou, E. Lesne, P. Sivakumar, C. Luo, F. Radu, P. Werner, C. Felser, and S. Parkin, Anomalous and topological Hall effects in epitaxial thin films of the noncollinear antiferromagnet $\mathrm{Mn}_{3}$ Sn, Phys. Rev. B 101, 094404 (2020).

[18] P. Brown, V. Nunez, F. Tasset, J. Forsyth, and P. Radhakrishna, Determination of the magnetic structure of $\mathrm{Mn}_{3} \mathrm{Sn}$ using generalized neutron polarization analysis, J. Phys.: Condens. Matter 2, 9409 (1990).

[19] J. Kübler and C. Felser, Weyl fermions in antiferromagnetic $\mathrm{Mn}_{3} \mathrm{Sn}$ and $\mathrm{Mn}_{3} \mathrm{Ge}$, Europhys. Lett. 120, 47002 (2018).

[20] H. Yang, Y. Sun, Y. Zhang, W.-J. Shi, S. S. P. Parkin, and B. Yan, Topological Weyl semimetals in the chiral antiferromagnetic materials $\mathrm{Mn}_{3} \mathrm{Ge}$ and $\mathrm{Mn}_{3} \mathrm{Sn}$, New J. Phys. 19, 015008 (2017). 
[21] K. Kuroda, T. Tomita, M.-T. Suzuki, C. Bareille, A. Nugroho, P. Goswami, M. Ochi, M. Ikhlas, M. Nakayama, S. Akebi et $a l$., Evidence for magnetic Weyl fermions in a correlated metal, Nat. Mater. 16, 1090 (2017).

[22] A. A. Burkov, Anomalous Hall Effect in Weyl Metals, Phys. Rev. Lett. 113, 187202 (2014).

[23] M. Ikhlas, T. Tomita, and S. Nakatsuji, Sample quality dependence of the magnetic properties in non-collinear antiferromagnet $\mathrm{Mn}_{3} \mathrm{Sn}$, JPS Conf. Proc. 30, 011177 (2020).

[24] W. J. Feng, D. Li, W. J. Ren, Y. B. Li, W. F. Li, J. Li, Y. Q. Zhang, and Z. D. Zhang, Glassy ferromagnetism in $\mathrm{Ni}_{3}$ Sn-type $\mathrm{Mn}_{3.1} \mathrm{Sn}_{0.9}$, Phys. Rev. B 73, 205105 (2006).

[25] S. Tomiyoshi, S. Abe, Y. Yamaguchi, H. Yamauchi, and H. Yamamoto, Triangular spin structure and weak ferromagnetism of $\mathrm{Mn}_{3} \mathrm{Sn}$ at low temperature, J. Magn. Magn. Mater. 54, 1001 (1986).

[26] N. H. Sung, F. Ronning, J. D. Thompson, and E. D. Bauer, Magnetic phase dependence of the anomalous Hall effect in $\mathrm{Mn}_{3} \mathrm{Sn}$ single crystals, Appl. Phys. Lett. 112, 132406 (2018).

[27] P. Park, J. Oh, K. Uhlirova, J. Jackson, A. Deak, L. Szunyogh, K. H. Lee, H. Cho, H.-L. Kim, H. C. Walker, D. Adroja, V. Sechovsky, and J.-G. Park, Magnetic excitations in non-collinear antiferromagnetic Weyl semimetal $\mathrm{Mn}_{3} \mathrm{Sn}$, npj Quantum Mater. 3, 63 (2018).

[28] P. E. Blöchl, Projector augmented-wave method, Phys. Rev. B 50, 17953 (1994).

[29] G. Kresse and J. Hafner, Ab initio molecular dynamics for liquid metals, Phys. Rev. B 47, 558 (1993).

[30] G. Kresse and J. Hafner, Ab initio molecular-dynamics simulation of the liquid-metal-amorphous-semiconductor transition in germanium, Phys. Rev. B 49, 14251 (1994).

[31] G. Kresse and J. Furthmüller, Efficient iterative schemes for $a b$ initio total-energy calculations using a plane-wave basis set, Phys. Rev. B 54, 11169 (1996).

[32] G. Kresse and J. Furthmüller, Efficiency of ab initio total energy calculations for metals and semiconductors using a plane-wave basis set, Comput. Mater. Sci. 6, 15 (1996)

[33] J. P. Perdew, K. Burke, and M. Ernzerhof, Generalized Gradient Approximation Made Simple, Phys. Rev. Lett. 77, 3865 (1996).

[34] FLEUR Project, http://www.flapw.de.

[35] P. Kurz, F. Förster, L. Nordström, G. Bihlmayer, and S. Blügel, $\mathrm{Ab}$ initio treatment of noncollinear magnets with the fullpotential linearized augmented plane wave method, Phys. Rev. B 69, 024415 (2004).
[36] T. Moriya, Anisotropic superexchange interaction and weak ferromagnetism, Phys. Rev. 120, 91 (1960).

[37] M. Ikhlas, T. Tomita, T. Koretsune, M.-T. Suzuki, D. NishioHamane, R. Arita, Y. Otani, and S. Nakatsuji, Large anomalous Nernst effect at room temperature in a chiral antiferromagnet, Nat. Phys. 13, 1085 (2017).

[38] B. Hardrat, A. Al-Zubi, P. Ferriani, S. Blügel, G. Bihlmayer, and S. Heinze, Complex magnetism of iron monolayers on hexagonal transition metal surfaces from first principles, Phys. Rev. B 79, 094411 (2009).

[39] A. K. Nandy, N. S. Kiselev, and S. Blügel, Interlayer Exchange Coupling: A General Scheme Turning Chiral Magnets into Magnetic Multilayers Carrying Atomic-Scale Skyrmions, Phys. Rev. Lett. 116, 177202 (2016).

[40] S. Nakamura, N. Kabeya, M. Kobayashi, K. Araki, K. Katoh, and $\mathrm{A}$. Ochiai, Spin trimer formation in the metallic compound $\mathrm{Gd}_{3} \mathrm{Ru}_{4} \mathrm{Al}_{12}$ with a distorted kagome lattice structure, Phys. Rev. B 98, 054410 (2018)

[41] K. Xu, Y. L. Zhang, Y. M. Cao, X. J. He, Z. Li, S. X Wei, Y. R. Kang, and C. Jing, Baromagnetic effect in the hexagonal $\mathrm{Mn}_{3} \mathrm{Sn}$ system, IEEE Trans. Magn. 53, 1 (2017).

[42] T. Nagamiya, S. Tomiyoshi, and Y. Yamaguchi, Triangular spin configuration and weak ferromagnetism of $\mathrm{Mn}_{3} \mathrm{Sn}$ and $\mathrm{Mn}_{3} \mathrm{Ge}$ Solid State Commun. 42, 385 (1982).

[43] Z. Q. Liu, H. Chen, J. M. Wang, J. H. Liu, K. Wang, Z. X. Feng, H. Yan, X. R. Wang, C. B. Jiang, J. M. D. Coey, and A. H. MacDonald, Electrical switching of the topological anomalous Hall effect in a non-collinear antiferromagnet above room temperature, Nat. Electron. 1, 172 (2018).

[44] R. Reis, M. Ghorbani Zavareh, M. Ajeesh, L. Kutelak, A. Sukhanov, S. Singh, J. Noky, Y. Sun, J. Fischer, K. Manna, C. Felser, and M. Nicklas, Pressure tuning of the anomalous Hall effect in the chiral antiferromagnet $\mathrm{Mn}_{3} \mathrm{Ge}$, Phys. Rev. Mater. 4, 051401 (2020).

[45] H. Tsai, T. Higo, K. Kondou, T. Nomoto, A. Sakai, A Kobayashi, T. Nakano, K. Yakushiji, R. Arita, S. Miwa, Y. Otani, and S. Nakatsuji, Electrical manipulation of a topological antiferromagnetic state, Nature (London) 580, 608 (2020).

[46] T. Matsumura, Y. Ozono, S. Nakamura, N. Kabeya, and A. Ochiai, Helical ordering of spin trimers in a distorted kagome lattice of $\mathrm{Gd}_{3} \mathrm{Ru}_{4} \mathrm{Al}_{12}$ studied by resonant x-ray diffraction, $\mathrm{J}$. Phys. Soc. Jpn. 88, 023704 (2019). 